\title{
A Stochastic Programming Model for the Thermal Optimal Day-Ahead Bid Problem with Physical Futures Contracts ${ }^{t / 2}$
}

\author{
Cristina Corchero $^{\text {a,* }}$, F.-Javier Heredia ${ }^{\mathrm{a}}$ \\ ${ }^{a}$ Department of Statistics and Operations Research, Universitat Politecnica Catalunya. Barcelona, Spain.
}

\begin{abstract}
The reorganization of the electricity industry in Spain completed a new step with the start-up of the Derivatives Market. One main characteristic of MIBEL's Derivatives Market is the existence of physical futures contracts; they imply the obligation to physically settle the energy. The market regulation establishes the mechanism for including those physical futures in the day-ahead bidding of the Generation Companies. The goal of this work is to optimize coordination between physical futures contracts and the day-ahead bidding which follow this regulation. We propose a stochastic quadratic mixed-integer programming model which maximizes the expected profits, taking into account futures contracts settlement. The model gives the simultaneous optimization for the Day-Ahead Market bidding strategy and power planning production (unit commitment) for the thermal units of a price-taker Generation Company. The uncertainty of the Day-Ahead Market price is included in the stochastic model through a set of scenarios. Implementation details and some first computational experiences for small real cases are presented.
\end{abstract}

Key words: Stochastic programming, OR in energy, electricity Day-Ahead Market, futures contracts, optimal bid

\section{Introduction}

Recently, there has been a reorganization of the electricity industry. The deregulation of the generation and distribution of electricity carried out in most countries in Europe has changed the problems that the generation companies (GenCo) have to face. With the introduction of the Electricity Markets, the price of electricity has become a significant risk factor. One of the techniques for hedging against market-price risk is participation in futures markets (Deng and Oren [1]) and, for this reason, the creation of Derivatives Electricity Markets has been a natural step in the deregulation process.

The most common mechanisms in the Electricity Markets are as follows:

- Out of the market products: the contracts that are traded directly between client and producer as, for example, bilateral contracts.

- Derivatives Market: pool where the medium term financial and physical derivatives products are traded; futures contracts, swap options and forward contracts can be negotiated in them.

- Day-Ahead Market: pool where the most of the physical production is traded. Seller and purchase agents submit their respective bids to the market operator who, the day before the delivery day, matches the bids.

- Intraday Markets: sequence of secondary markets that are used (1) by the system operator to guarantee the reliability of the system and (2) by the producers and consumers to change the result of the Day-Ahead Market.

\footnotetext{
The work of C. Corchero was supported by FPI Grant BES-2006-12311, and the work of F.-Javier Heredia was partially supported by Grants DPI2005-09117-C02-01 and DPI2008-02153, all of them grants of the Ministry of Science and Innovation of Spain

${ }^{*}$ Corresponding author. Address: Ed. C5-201, Campus Nord, Jordi Girona 1-3 08034 Barcelona. Spain. Tel.: +34-934-016-939. Fax: $+34-934-015-855$

Email addresses: cristina. corchero@upc.edu (Cristina Corchero), f.javier.heredia@upc. edu (F.-Javier Heredia) 
In this paper, we will focus on the Spanish Electricity Market but as a particular example of many other similar deregulations that have been carried out all over the world. The models presented in this work can be easily applied or adapted to many others Electricity Markets that contain physical derivatives products and Day-Ahead Market auctions.

On the Spanish mainland, the Electricity Market, which was launched in 1998, includes a Day-Ahead Market and a set of balancing and adjustment markets. As the introduction of competition and the deregulation process did not behave as expected, the Spanish Market was improved in 2007 with the start-up of the Iberian Electricity Market (MIBEL) and some other new regulations. The MIBEL brings together the Spanish and Portuguese electricity systems and it complements the previous Spanish Electricity Market with a Derivatives Market. Generation companies can no longer optimize their short-term generation planning decisions without considering the relationship between those markets. The short-term bidding strategies are based on the GenCo's day-ahead bid, which is defined as the selling offer submitted by a GenCo to the Day-Ahead Market operator. It is always a non-decreasing step-wise function giving the price at which the GenCo offers its electricity generation to the pool.

There are two main derivatives physical products, the bilateral contracts (BC) and the futures contracts (FC). The $\mathrm{BCs}$ are agreements between a generation company and a qualified consumer to provide a given amount of electrical energy at a stipulated price in a delivering period. The characteristics of the BCs (energy, price and delivering period) are negotiated between the two parts before the Day-Ahead Market, the MIBEL's rules state that each GenCo must notify the scheduling of the BCs to the system and market operator before the closure of the Day-Ahead Market.

The FCs is an medium-term exchange-traded derivative that represents agreements to buy/sell some underlying asset in the future at a specified price. The main characteristics of a FCs are the asset, the contract size, the delivery arrangements and period (weeks, months, quarters or years), and the price. In the MIBEL, the FCs are offered through the Derivatives Market, an average of $2340 \mathrm{GWh}$ are traded monthly. In contrast to other Electricity Derivatives Markets, the delivery arrangements of the MIBEL FCs offer a choice between a physical or financial settlement. Physical futures contracts have cash settlement and physical delivery whereas financial contracts have cash settlement only. This physical delivery option is the feature of the FCs that interacts with the GenCo day-ahead bidding process (OMEL [2]). Thus, although the physical FC is a medium-term product, the energy amount of this FC has to be physically delivered daily, and included in the day-ahead bid of the GenCo, coupling this way the medium and short term.

The main differences between the BCs and the FCs is that the first ones are traded between the GenCo and the consumer out of the market meanwhile the FCs are traded at the Derivatives Market. Moreover, the quantity committed at the BCs is not bid to the Day-Ahead Market while the MIBEL rules forces the quantity committed at FCs to be bid to the Day-Ahead Market through a sale offer with a bid price of $0 € / \mathrm{MWh}$. Among this derivatives products, we will focus on the physical futures contracts.

In liberalized electricity markets, a GenCo must build an hourly bid that is sent to the market operator, who selects the lowest price among the bidding companies in order to match the demand. Some earlier studies give the optimal bidding quantity once the expected distribution of the spot prices is known (Shrestha et al. [3], Triki et al. [4], Musmanno et al. [5]) but do not propose any explicit modelization of the optimal bid. Conejo et al. [6] proposes an optimal stepwise bidding strategy for a price-taker GenCo based on the units characteristics, the expected spot price, and the optimal generation. Furthermore, Gountis and Bakirtzis [7] considers the approximation of stepwise bid curves by linear bid functions based on the marginal costs and the optimal generation quantity. Nabona and Pages [8] gives a three stage procedure to build the optimal bid based on the optimal generation quantity and the zero-price bid. Also, Ni et al. [9] uses the concept of price-power function, which is similar to the matched energy function used in our work, to derive the optimal bid curves of a hydro-thermal system. Nowak et al. [10] and Fleten and Kristoffersen [11] also distinguish between the variables representing the bid energy and those corresponding to the matched energy in the case of a price-taker GenCo. In particular, Fleten and Kristoffersen [11] has some aspects that are very related to this work; it presents a stochastic programming model to optimize the unit commitment and the day-ahead bidding of a hydropower producer in the Nord Pool. Moreover, general considerations about optimal bidding construction in electricity markets can be obtained in Anderson and Philpott [12] and Anderson and Xu [13]. Neither of these studies mentioned includes FCs.

Some different approaches to the inclusion of FCs in the management of a GenCo can be found in the electricity market literature. Most of the literature defines forward contracts as contracts with a physical settlement and futures contracts as contracts with a financial settlement. The main theoretical differences between these two kinds of derivatives products is the level of standardization and the kind of market where they are traded (Hull [14]). We focus on 
the inclusion of physical derivatives products in the short-term management of a GenCo, other general considerations about FCs can be found in many works, for instance, Hull [14], Collins [15], Neuberger [16] or Carlton [17].

Prior to deregulation, Kaye et al. [18] illustrates how physical and financial contracts can be used to hedge against the risk of profit volatility, allowing for flexible responses to spot price. After Day-Ahead and Derivatives Markets start-up, Bjorgan et al. [19] presents a theoretical framework for the integration of FCs into the risk management of a GenCo. Also, Chen et al. [20] presents a bidding decision making system for a GenCo taking into account the impacts of several types of physical and financial contracts; this system is based on a market-oriented unit commitment model, a probabilistic local marginal price simulator, and a multi-criteria decision system. Furthermore, Conejo et al. [21] optimizes the forward physical contracts portfolio up to one year, taking into account the day-ahead bidding; the objective of the study is to protect against the pool price volatility through FCs. Moreover, Guan et al. [22] optimizes in a medium-term horizon the generation asset allocation between different derivatives products and the spot market, taking into account short-term operating constraints; it considers the known price of the contracts and forecasts the spot price. In a different framework, Musmanno et al. [5] considers the Italian bilateral contracts in a similar way to ours FCs but without developing an explicit bidding function. Once again, neither of this studies proposes a explicit bidding function that coordinates the day-ahead bidding with the economic dispatch of the futures contracts. From another point of view, Tanlapco et al. [23] does a statistical study of the reduction in risk due to forward contracts; it is shown that, for a GenCo, the electricity FCs are better to hedge price risk than other related futures as crude oil or gas futures contracts.

As stated above, we are dealing with a new electricity futures contract situation due to the MIBEL definition of physical FCs, hence, as far as we know, there is no previous work dealing with the short-term management of the GenCo which includes the coordination between day-ahead bidding strategies and physical futures settlement following the Iberian Market rules. The MIBEL regulation (OMEL [2]) describes the coordination between this physical FCs portfolio and the day-ahead bidding mechanism of the GenCo. That regulation obliges the GenCo to determine its generation scheduling in order to be able to cover those obligations and to determine its optimal offer, taking into account those FCs. Following the idea that the participation in the Spot and the Derivatives Markets has to be studied jointly, the main objective of this work is to build a stochastic programming model which includes the coordination between physical FCs and Day-Ahead Market bidding following the MIBEL rules. In other words, we want to see how the inclusion of FCs in the model affects the short term bidding strategies of the GenCo in the Day-Ahead Market.

The main contributions of this paper are as follows:

- A new quadratic mixed-integer stochastic programming model, for the optimal day-ahead bid with Future Contracts problem (DABFC) that maximize the benefits arising from the Day-Ahead Market with the integration of the energy of the Physical Futures Contract in the Day-Ahead Market.

- A theoretical study of the solutions of the DABFC model that provides, for the very first time, the analytical expression of the optimal bid functions that ensures the maximization of the long run expected benefits of those GenCos that participates in the Day-Ahead Market having FC obligations.

- A detailed validation of the DABFC model with real data coming from the MIBEL Iberian Electricity Market showing the influence of the physical Futures Contracts in the GenCo's optimal bidding strategy.

In section 2, the stochastic programming model for the coordination between day-ahead bidding and the physical futures contract portfolio -taking into account thermal unit operational constraints- is presented. In section 3 , the optimal bid function is developed and its properties are described. In section 4, a detailed case study is solved and analyzed. Finally, in section 5, some relevant conclusions are presented.

\section{Model}

\subsection{Coordination between Day-Ahead and Derivatives Markets}

As stated above, the MIBEL regulation OMEL [2] describes the coordination between the physical futures contracts portfolio and the day-ahead bidding mechanism (Fig. 1), i.e. how to include the result of the medium-term physical products into the short-term bidding strategies. This coordination is structured in the following three phases: 


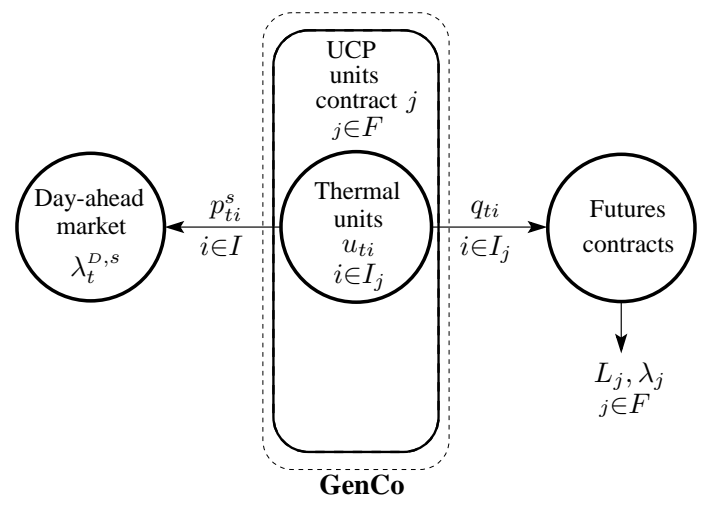

Figure 1: Representation of the system under study at period $t$

1. For every derivatives contract in which the GenCo is interested, it has to define the Term Contract Units (UCP in the MIBEL's notation) which are virtual units allowed to be offered in the Derivatives Market. Each UCP is formed by the subset of the physical units of the GenCo which will generate the energy to cover the corresponding contract. For each contract, a physical unit can only participate in one virtual UCP.

2. Two days before the delivery date the GenCo receives from the Derivatives Market Operator, OMIP [24] the quantity that every UCP has to produce in order to cover the matched FCs. This information is also sent to the Day-Ahead Market Operator, OMEL [25].

3. OMEL demands that every GenCo commits the quantity designated to FCs through the Day-Ahead Market bidding of the physical units that form each UCP. This commitment is made by the so called instrumental price offer, that is, a sale offer with a bid price of $0 € / \mathrm{MWh}$ (also called price acceptant). It is noteworthy that this is the main difference between FCs and BCs because, contrary to the FCs, the energy committed at the BCs must be excluded from the MIBEL's Day-Ahead Market bid.

That regulation implies that the GenCo has to determine its unit commitment in order to be able to cover those obligations and it has to determine its optimal bid by taking into account those instrumental price offers. Due to the algorithm the market operator uses to clear the Day-Ahead Market, all instrumental price offers will be matched (i.e. accepted) in the clearing process, that is, this energy shall be produced and will be remunerated at the spot price.

Following MIBEL's rules, if we are optimizing today we focus on tomorrow's Day-Ahead Market because we have to submit tomorrow's bidding. Thus, the optimization horizon is at 24-hour intervals; this set of intervals is denoted as $T$. The proposed short-term bidding strategies are addressed to a price-taker GenCo. The generation units to be considered are the thermal units with participation in the auction process; these thermal units have a quadratic cost function that defines the relationship between the energy generation (MWh) and the fuel cost. Thus, the relevant parameters of a thermal unit are:

- quadratic generation costs with constant, linear and quadratic coefficients, $c_{i}^{b}(€), c_{i}^{l}(€ / \mathrm{MWh})$ and $c_{i}^{q}(€ / \mathrm{MWh})^{2}$ respectively, for the $i^{\text {th }}$ unit.

- $\bar{P}_{i}$ and $\underline{P}_{i}$ the upper and lower bound, respectively, on the energy generation (MWh) of a committed unit $i$.

- start-up, $c_{i}^{\text {on }}$, and shut-down, $c_{i}^{\text {off }}$, costs $(€)$ for the $i^{\text {th }}$ unit.

- minimum operation and minimum idle time, $t_{i}^{o n}$ and $t_{i}^{o f f}$ respectively, for the $i^{t h}$ unit., i.e., the minimum number of hours that the unit must remain in operation once it is started up and the minimum number of hours that the unit must remain idle once it has been shut down before being started up again, respectively.

\subsection{First stage binary variables and thermal units operation constraints}

The formulation of the start-up and shut-down processes follows Nabona and Pages [8]. Let $u_{t i} \in\{0,1\}$ be a firststage binary variable expressing the off-on operating status of the $i^{\text {th }}$ unit over the $t^{\text {th }}$ interval $\left(u_{t i}=1\right.$ if committed, 
$u_{t i}=0$ if uncommitted). The values of $u_{t i}$ and $u_{(t+1) i}$ must obey certain operating rules in order to take into account the constraints of the minimum in service and idle time. It is necessary to introduce two extra binary variables $e_{t i}$ and $a_{t i}$ for each $u_{t i}$. Let $e_{t i} \in\{0,1\}$ be a start-up indicator for the $i^{t h}$ unit. It has a value of one in all intervals $t$ where the $i^{t h}$ unit has changed from $u_{(t-1) i}=0$ to $u_{t i}=1$, and zero elsewhere. Similarly, $a_{t i} \in\{0,1\}$ is a shut-down indicator for the $i^{t h}$ unit. It should have a value of one in all intervals $t$ where $u_{(t-1) i}=1$ to $u_{t i}=0$, and zero otherwise. The following three sets of constraints unambiguously model the commitment variable $u_{t i}$ and the start-up and shut-down variables $e_{t i}$ and $a_{t i}$ :

$$
\begin{array}{cl}
u_{t i}-u_{(t-1) i}-e_{t i}+a_{t i}=0 & \forall i \in I, \forall t \in T \\
e_{t i}+\sum_{k=t}^{\min \left\{t+t_{i}^{o n},|T|\right\}} a_{k i} \leq 1 & \forall i \in I, \forall t \in T \\
a_{t i}+\sum_{k=t+1}^{\min \left\{t+t_{i}^{o f f},|T|\right\}} e_{k i} \leq 1 & \forall i \in I, \forall t \in T
\end{array}
$$

\subsection{First stage continuous variables and futures contracts covering constraints}

Let $q_{t i}$ be the first-stage variable standing for the energy of the instrumental price offer, that is, the energy bid by unit $i$ to the $t^{\text {th }}$ Day-Ahead Market at $0 € / M W h$. If variable $f_{\text {tij }}$ represents the energy of the $j^{\text {th }}$ FC allocated to thermal unit $i$ at period $t$, then the following constraints must be satisfied:

$$
\begin{array}{clrl}
\sum_{i \in I_{j}} f_{t i j} & =L_{j} & & \forall t \in T, \forall j \in F \\
q_{t i} & \geq \sum_{\forall j \in F_{i}} f_{t i j} & & \forall i \in I \quad \forall t \in T \\
\underline{P}_{i} u_{t i} \leq q_{t i} \leq \bar{P}_{i} u_{t i} & & \forall i \in I \quad \forall t \in T \\
f_{t i j} & \geq 0 & & \forall i \in I, \forall t \in T, \forall j \in F
\end{array}
$$

where the known parameters $F_{i}, I_{j}$ and $L_{j}$ are, respectively, the subset of contracts in which unit $i$ participates, the set of thermal units that participates in contract $j$ (the units in all the UCPs that participate in the contract $j$ ) and the energy that has to be settled for contract $j$. Constraint (4) ensures that the energy of the $j^{\text {th }}$ futures contracts $L_{j}$ will be completely dispatched among all the committed units of its associated UCPs. Constraints (5) formulate the MIBEL's rule that forces the energy of the future contracts to be bid through the instrumental price offer, that is to say, the variable $q_{t i}$ represents the quantity of the instrumental price bid and it must be not less than the sum of the energy allocated to FCs. The lower bound $q_{t i} \geq \underline{P}_{i} u_{t i}$ prevents committed thermal units from being matched below their minimum generation limit meanwhile the upper bound $q_{t i} \leq \bar{P}_{i} u_{t i}$ prevents production levels above the operational limit (6). It is interesting to remark that if we include BC instead of FC, only the constraint (4) will remain unchanged while all other constraints and the results presented in Section 3 will change. All this differences between the modelization of FCs and BCs are due to the specific MIBEL rules for each product.

\subsection{Second stage variables: matched energy}

The formulation of the objective function of the present model will include variables representing the value of the matched energy for the committed thermal unit $i$ on the $t^{t h}$ Day-Ahead Market. For the moment, the matched energy will be loosely defined as the accepted energy in the clearing process; that is, the energy that the thermal $i$ should generate at period $t$ and that will be rewarded at the clearing price. This matched energy, which plays a central role in our model, is uniquely determined by the sale bid and the clearing price. A sale bid in the MIBEL's Day-Ahead Market consists of a stepwise non-decreasing curve defined by up to 10 energy (MWh)-price( $€ /$ MWh) blocks. As usual in this kind of work (see Gountis and Bakirtzis [7]) we will consider a simplified modelization of the true sale bid through the so called bid function $\lambda_{t i}^{b}$, not necessarily stepwise: 
Definition 1 (Bid function). A bid function for the thermal unit $i$ is a non-decreasing function defined over the interval $\left[0, \bar{P}_{i}\right]$ that gives, for any feasible value of the bid energy $p_{t i}^{b}$, the asked price per MWh from the Day-Ahead Market:

$$
\begin{aligned}
\lambda_{t i}^{b}: \quad\left[0, \bar{P}_{i}\right] & \longrightarrow \Re^{+} \cup 0 \\
p_{t i}^{b} & \longmapsto \lambda_{t i}^{b}\left(p_{t i}^{b}\right)
\end{aligned}
$$

For a given bid function $\lambda_{t i}^{b}$ the matched energy associated with the clearing price $\lambda_{t}^{d}, p_{t i}^{m}$ is defined through the matched energy function

Definition 2 (Matched energy function). The matched energy associated with the bid function $\lambda_{t i}^{b}$ is defined as the maximum bid energy with an asked price not greater than the clearing price $\lambda_{t}^{d}$, and is represented by the function:

$$
p_{t i}^{m}\left(\lambda_{t}^{d}\right) \stackrel{\text { def }}{=} \max \left\{p_{t i}^{b} \in\left[0, \bar{P}_{i}\right] \mid \lambda_{t i}^{b}\left(p_{t i}^{b}\right) \leq \lambda_{t}^{d}\right\}
$$

The clearing price $\lambda_{t}^{d}$ is a random variable that will be modeled through a set of scenarios $S$ with associated spot prices $\lambda^{d, s}=\left\{\lambda_{1}^{d, s}, \ldots, \lambda_{T}^{d, s}\right\}$ and probabilities $P^{s}=P\left(\lambda^{d, s}\right), s \in S$. Each one of these scenarios has, for each period $t$, a corresponding matched energy that will be represented in the model by the second stage variable $p_{t i}^{s}$. Although our model will be developed without any assumption on the specific expression of the bid function $\lambda_{t i}^{b}$ it is necessary, for the sake of the model's consistency, to assume the existence of a bid function with a matched energy function (9) that agrees with the optimal value of variables $p_{t i}^{s}$, i.e.:

Assumption 1. For any thermal unit $i$ committed at period t there exists a bid function $\lambda_{t i}^{b}$ such that:

$$
p_{t i}^{m}\left(\lambda_{t}^{d, s}\right)=p_{t i}^{s *} \quad \forall s \in S
$$

with $p_{t i}^{s *}$ the optimal value of variable $p_{t i}^{s}$

Notice that the existence of such a bid function is not evident, as all scenarios must prove simultaneously equal (10). In Section 3 it will be proved that a bid function $\lambda_{t i}^{b}$ satisfying (10) (optimal bid) always exist.

The matched energy $p_{t i}^{s}$ is related to the rest of the first stage variable through the following set of constraints:

$$
\begin{array}{ll}
p_{t i}^{s} \leq \bar{P}_{i} u_{t i} & \forall i \in I, \forall t \in T, \forall s \in S \\
p_{t i}^{s} \geq q_{t i} & \forall i \in I, \forall t \in T, \forall s \in S \\
q_{t i} \geq \underline{P}_{i} u_{t i} & \forall i \in I, \forall t \in T
\end{array}
$$

This set of constraints substitutes the bounds on $q_{t i}$ defined in (6).

\subsection{Objective function}

The expected value of the benefit function $B$ can be expressed as:

$$
\begin{aligned}
E_{\lambda^{d}}[B(u, a, e, p)]= & \sum_{\forall t \in T \forall j \in F}\left(\lambda_{j}^{f}-\bar{\lambda}_{t}^{d}\right) L_{j} \\
& -\sum_{\forall t \in T \forall i \in I} \sum_{\forall}\left[c_{i}^{o n} e_{t i}+c_{i}^{o f f} a_{t i}\right] \\
& +\sum_{\forall t \in T} \sum_{\forall i \in I \forall s \in S} \sum^{s}\left[\lambda_{t}^{d, s} p_{t i}^{s}-\left(c_{i}^{b} u_{t i}+c_{i}^{l} p_{t i}^{s}+c_{i}^{q}\left(p_{t i}^{s}\right)^{2}\right)\right]
\end{aligned}
$$

where:

(14) is a constant term, which would be excluded from the optimization, and corresponds to the incomes of the FCs, which are settled by differences. $\lambda_{j}^{f}$ represents the futures settlement price and $\bar{\lambda}_{t}^{d}=\sum_{s \in S} P^{s} \lambda_{t}^{d, s}$ is the mean of the Day-Ahead Market price scenarios. 
(15) is the on/off fixed cost of the unit commitment of the thermal units. This term is deterministic and does not depend on the realization of the random variable $\lambda_{t}^{d}$.

(16) represents the expected value of the benefit from the Day-Ahead Market, where $P^{s}$ is the probability of scenario $s$. The first term, $\lambda_{t}^{d, s} p_{t i}^{s}$, computes the incomes from the Day-Ahead Market due to a value $p_{t i}^{s}$ of the matched energy, while the term between parentheses corresponds to the expression of the quadratic generation costs. Of course, $c_{i}^{b} u_{t i}$ could have been added to the deterministic term (15), as it doesn't depend on the scenario, but it has been conserved in (16) for the sake of clarity.

All the functions appearing in Eqs. (15) and (16) are linear except the term (16), which is concave quadratic $\left(c_{i}^{q} \geq 0\right.$, see Table 4.1).

\subsection{The Day-Ahead Bid with Futures Contracts problem}

The full model developed in the preceding sections, the so-called Day-Ahead Bid with Futures Contracts problem can be formulated as:

$$
\begin{array}{ll}
\text { (DABFC) } & \\
\operatorname{minimize}_{p, q, f, a, e, u} \sum_{\forall t \in T} \sum_{\forall i \in I}\left(c_{i}^{o n} e_{t i}+c_{i}^{o f f} a_{t i}+c_{i}^{b} u_{t i}+\sum_{s \in S} P^{s}\left[\left(c_{i}^{l}-\lambda_{t}^{d, s}\right) p_{t i}^{s}+c_{i}^{q}\left(p_{t i}^{s}\right)^{2}\right]\right) \\
\text { s.t. } & \forall t \in T, \forall j \in F \\
\sum_{i \in I_{j}} f_{t i j}=L_{j} & \forall i \in I, \forall t \in T \\
q_{t i} \geq \sum_{j \in F_{i}} f_{t i j} & \forall i \in I, \forall t \in T \\
u_{t i}-u_{(t-1) i}-e_{t i}+a_{t i}=0 & \forall i \in I, \forall t \in T \\
e_{t i}+\sum_{k=t}^{\min \left\{t+t_{i}^{n},|T|\right\}} a_{k i} \leq 1 & \\
\quad \min _{\left.k t+t_{i}^{o f f},|T|\right\}} e_{k i} \leq 1 & \forall i \in I, \forall t \in T \\
a_{t i}+\sum_{k=t+1}^{s} e_{t i}^{s} \leq \bar{P}_{i} u_{t i} & \forall i \in I, \forall t \in T, \forall s \in S \\
p_{t i}^{s} \geq q_{t i} & \forall i \in I, \forall t \in T, \forall s \in S \\
q_{t i} \geq \underline{P}_{i} u_{t i} & \forall i \in I, \forall t \in T \\
f_{t i j} \geq 0 & \forall i \in I, \forall t \in T, \forall j \in F \\
u_{t i}, a_{t i}, e_{t i} \in\{0,1\} & \forall i \in I, \forall t \in T
\end{array}
$$

This formulation corresponds to a mixed continuous binary linearly constrained minimization problem with concave quadratic objective function. In the next sections the properties of the optimal solutions of the (DABFC) problem will be studied.

\section{Optimal Bid}

The preceding model (17)-(27) is built on the assumption 1, which presumes the existence of a bid function $\lambda_{t i}^{b}$ with a matched energy function consistent with the optimal solution of the (DABFC) problem, i.e.:

$$
p_{t i}^{m}\left(\lambda_{t}^{d, s}\right)=p_{t i}^{s *} \quad \forall s \in S
$$

The objective of this section is the development of such a bid function, called the optimal bid function $\lambda_{t i}^{b *}\left(p_{t i}^{b}\right)$. In order to derive this optimal bid function, the properties of the optimal solutions of the problem (17)-(27) will be studied in the next section and used to derive the expression of the optimal matched energy $p_{t i}^{s *}$ in terms of the instrumental energy bid $q_{t i}^{*}$. 


\subsection{Optimal matched energy}

Let $x^{* \prime}=\left[u^{*}, a^{*}, e^{*}, p^{*}, q^{*}, f^{*}\right]^{\prime}$ represent the optimal solution of the (DABFC) problem. Fixing the binary variables to its optimal value $u^{*}, a^{*}$ and $e^{*}$ in the formulation of the (DABFC) problem, we obtain the following convex quadratic continuous problem:

$$
\begin{aligned}
& \left(\mathrm{DABFC}^{*}\right): \\
& \underset{p, q, f}{\operatorname{minimize}} \sum_{\forall t \in T} \sum_{\forall i \in I_{o n_{t}}^{*} \in S} \sum_{s \in S} P^{s}\left[\left(c_{i}^{l}-\lambda_{t}^{d, s}\right) p_{t i}^{s}+c_{i}^{q}\left(p_{t i}^{s}\right)^{2}\right] \\
& \text { s.t. } \\
& \sum_{i \mid i \in I_{j} \cap I_{\text {ont }}^{*}} f_{t i j}=L_{j} \quad \forall t \in T, \forall j \in F \\
& q_{t i} \geq \sum_{j \in F_{i}} f_{t i j} \quad \forall t \in T, \forall i \in I_{o n_{t}}^{*} \\
& p_{t i}^{s} \leq \bar{P}_{i} \quad \forall t \in T, \forall i \in I_{o n_{t}}^{*}, \forall s \in S \\
& p_{t i}^{s} \geq q_{t i} \quad \forall t \in T, \forall i \in I_{o n_{t}}^{*}, \forall s \in S \\
& q_{t i} \geq \underline{P}_{i} \quad \forall t \in T, \forall i \in I_{o n_{t}}^{*} \\
& f_{t i j} \geq 0 \quad \forall t \in T, \forall i \in I_{o n_{t}}^{*}, \forall j \in F
\end{aligned}
$$

with $I_{o n_{t}}^{*}:=\left\{i \in I \mid u_{t i}^{*}=1\right\}$, the set of thermal units committed at time "t". Obviously, the optimal solution of this continuous problem should coincide with the optimal value of the continuous variables of the original (DABFC) problem, $p^{*}, q^{*}$ and $f^{*}$. The (DABFC ${ }^{*}$ ) problem is separable by intervals, being the problem associated with the $t^{\text {th }}$ time interval in standard form (Luenberger [26]):

$$
\begin{array}{ll}
\left(\mathrm{DABFC}_{t}^{*}\right): & \\
\underset{p_{t}, q_{t}, f_{t}}{\operatorname{minimize}} \sum_{\forall i \in I_{o n_{t}}^{*}} \sum_{s \in S} P^{s}\left[\left(c_{i}^{l}-\lambda_{t}^{d, s}\right) p_{t i}^{s}+c_{i}^{q}\left(p_{t i}^{s}\right)^{2}\right] \\
\sum_{i \mid i \in I_{j} \cap I_{o n_{t}}^{*}} f_{t i j}-L_{j}=0 & \forall j \in F \\
\sum_{j \in F_{i}} f_{t i j}-q_{t i} \leq 0 & \forall i \in I_{o n_{t}}^{*} \\
p_{t i}^{s}-\bar{P}_{i} \leq 0 & \forall i \in I_{o n_{t}}^{*}, \forall s \in S \\
q_{t i}-p_{t i}^{s} \leq 0 & \forall i \in I_{o n_{t}}^{*}, \forall s \in S \\
\underline{P}_{i}-q_{t i} \leq 0 & \forall i \in I_{o n_{t}}^{*} \\
-f_{t i j} \leq 0 & \forall i \in I_{o n_{t}}^{*}, \forall j \in F
\end{array}
$$

where $\pi, \tilde{\mu}, \bar{\mu}, \hat{\mu}, \mu$ and $\breve{\mu}$ represent the Lagrange multiplier associated with each constraint.

The Karush-Kuhn-Tucker conditions of the $\left(\mathrm{DABFC}_{t}^{*}\right)$ problem can be expressed as: 


$$
\begin{array}{rll}
P^{s}\left[\left(c_{i}^{l}-\lambda_{t}^{d, s}\right)+2 c_{i}^{q} p_{t i}^{s *}\right]+ & \\
+\bar{\mu}_{t i}^{s}-\hat{\mu}_{t i}^{s}=0 & \forall i \in I_{o n_{t}}^{*}, \forall s \in S \\
-\tilde{\mu}_{t i}-\underline{\mu}_{t i}+\sum_{\forall s \in S} \hat{\mu}_{t i}^{s}=0 & \forall i \in I_{o n_{t}}^{*} \\
\tilde{\mu}_{t i}+\pi_{t j}-\breve{\mu}_{t i j}=0 & \forall i \in I_{o n_{t}}^{*}, \forall j \in F_{i} \\
\tilde{\mu}_{t i}\left(\sum_{j \in F_{i}} f_{t i j}-q_{t i}^{*}\right)=0 & \forall i \in I_{o n_{t}}^{*} \\
\bar{\mu}_{t i}^{s}\left(p_{t i}^{s *}-\bar{P}_{i}\right)=0 & \forall i \in I_{o n_{t}}^{*}, \forall s \in S \\
\underline{\mu}_{t i}\left(\underline{P}_{i}-q_{t i}^{*}\right)=0 & \forall i \in I_{o n_{t}}^{*} \\
\hat{\mu}_{t i}^{s}\left(q_{t i}^{*}-p_{t i}^{s *}\right)=0 & \forall i \in I_{o n_{t}}^{*}, \forall s \in S \\
\breve{\mu}_{t i j} f_{t i j}^{*}=0 & \forall i \in I_{o n_{t}}^{*}, \forall j \in F \\
\tilde{\mu}_{t i}, \bar{\mu}_{t i}^{s}, \underline{\mu}_{t i}, \hat{\mu}_{t i}^{s}, \breve{\mu}_{t i j} \geq 0 & \forall i \in I_{o n_{t}}^{*}, \forall j \in F, \forall s \in S
\end{array}
$$

The $\left(\mathrm{DABFC}_{t}^{*}\right)$ problem is convex $\left(c_{i}^{q} \geq 0\right)$ and then the system (43)-(51) represents the necessary and sufficient optimality conditions of the $\left(\mathrm{DABFC}_{t}^{*}\right)$ problem and, consequently, of the $\left(\mathrm{DABFC}^{*}\right)$ problem. Therefore the solution set of the preceding KKT system defines the value of variables $p_{t i}^{s}, q_{t i}$ and $f_{t i j}$ over the optimal solution of the (DABFC) problem associated with $I_{o n_{t}}^{*}$. The following lemma states this result:

Lemma 1. Let $x^{* \prime}=\left[u^{*}, a^{*}, e^{*}, p^{*}, q^{*}, f^{*}\right]^{\prime}$ be an optimal solution of the (DABFC) problem. Then, for any $x^{*}$ there exists Lagrange multipliers, $\tilde{\mu}, \bar{\mu}, \hat{\mu}, \underline{\mu}$ and $\breve{\mu}$ such that the value of variables $p^{*}, q^{*}$ and $f^{*}$ satisfy the KKT system (43)-(51). Conversely, for any solution $p^{*}, q^{*}$ and $f^{*}$ of the KKT system (43)-(51) associated with $I_{\text {ont }}^{*}$ the correspondent solution $x^{*}$ is optimal for the (DABFC) problem.

The fact that any solution of the (DABFC) problem must satisfy the system (43)-(51) will be exploited in the next two lemmas to derive the expressions of the optimal matched energy associated with scenario $s$ :

Lemma 2 (Optimal matched energy, quadratic costs). Let $x^{*}$ be an optimal solution of the (DABFC) problem. Then, for any unit $i$ with quadratic convex generation cost (i.e. $c_{i}^{q}>0$ ) committed at period $t$ (i.e. $i \in I_{o n_{t}}^{*}$ ), the optimal value of the matched energy $p_{t i}^{s *}$ can be expressed as:

$$
p_{t i}^{s *}=\max \left\{q_{t i}^{*}, p_{t i}^{d, s}\right\}
$$

where $p_{t i}^{d, s}$ is the constant parameter

$$
p_{t i}^{d, s}= \begin{cases}\underline{P}_{i} & \text { if } \theta_{t i}^{s} \leq \underline{P}_{i} \\ \theta_{t i}^{s} & \text { if } \underline{P}_{i} \leq \theta_{t i}^{s} \leq \bar{P}_{i} \\ \bar{P}_{i} & \text { if } \theta_{t i}^{s} \geq \bar{P}_{i}\end{cases}
$$

with

$$
\theta_{t i}^{s}=\left(\lambda_{t}^{d, s}-c_{i}^{l}\right) / 2 c_{i}^{q}
$$

Proof As lemma 1 establishes, any optimal solution of the (DABFC) problem must satisfy the KKT system (43)-(51). As $c_{i}^{q}>0$, equation (43) allows variable $p_{t i}^{s *}$ to be expressed as:

$$
p_{t i}^{s *}=\frac{\lambda_{t}^{d, s}-c_{i}^{l}}{2 c_{i}^{q}}+\frac{\hat{\mu}_{t i}^{s}-\bar{\mu}_{t i}^{s}}{2 c_{i}^{q} P^{s}}=\theta_{t i}^{s}+\frac{\hat{\mu}_{t i}^{s}-\bar{\mu}_{t i}^{s}}{2 c_{i}^{q} P^{s}}
$$

Equations (39)-(42) establishes that any optimal solution $x^{*}$ of the (DABFC) problem must satisfy that

$$
\underline{P}_{i} \leq q_{t i}^{*} \leq p_{9}^{s *} \leq \bar{P}_{i}
$$


To derive the relationships (52), the solution of the KKT system will be analyzed in the five cases among which any optimal solution of the (DABFC) problem could be classified according to (56). The rationale of the demonstration is to proof that, in all cases, the expression of the variable $p_{t i}^{s *}$ derived from the KKT system (43)-(51) coincides with (52):

(a) $\underline{P}_{i}<q_{t i}^{*}=p_{t i}^{s *}=\bar{P}_{i}$ : This is a trivial case, because, by definition (53), $p_{t i}^{d, s} \leq \bar{P}_{i}$, and then $p_{t i}^{s *}=\max \left\{q_{t i}^{*}=\right.$ $\left.\bar{P}_{i}, p_{t i}^{d, s} \leq \bar{P}_{i}\right\}=\bar{P}_{i}$

(b) $\underline{P}_{i} \leq q_{t i}^{*}<p_{t i}^{s *}=\bar{P}_{i}$ : Condition (49) gives $\hat{\mu}_{t i}^{s}=0$ that, together with the non-negativity of the lagrange multipliers $\bar{\mu}_{t i}^{s}$ and equation (55) sets $\bar{P}_{i} \leq \theta_{t i}^{s}$ and, by definition (53), $p_{t i}^{d, s}=\bar{P}_{i}$. Then $p_{t i}^{s *}=\max \left\{q_{t i}^{*}<\bar{P}_{i}, p_{t i}^{d, s}=\bar{P}_{i}\right\}=\bar{P}_{i}$

(c) $\underline{P}_{i} \leq q_{t i}^{*}<p_{t i}^{s *}<\bar{P}_{i}$ : In this case, conditions (47) and (49) give $\bar{\mu}_{t i}^{s}=\hat{\mu}_{t i}^{s}=0$, that, together with equation (55) gives $p_{t i}^{s *}=\theta_{t i}^{s}$. Then, as it is assumed that $\underline{P}_{i}<p_{t i}^{s *}<\bar{P}_{i}$, so is $\theta_{t i}^{s}$ and, by definition (53), $p_{t i}^{d, s}=\theta_{t i}^{s}=p_{t i}^{s *}>q_{t i}^{*}$. Therefore $p_{t i}^{s *}=\max \left\{q_{t i}^{*}, p_{t i}^{d, s}=\theta_{t i}^{s}>q_{t i}^{*}\right\}=p_{t i}^{d, s}$

(d) $\underline{P}_{i}<q_{t i}^{*}=p_{t i}^{s *}<\bar{P}_{i}$ : In this case, condition (47) forces $\bar{\mu}_{t i}^{s}=0$ which, in combination with equation (55) and condition $\hat{\mu}_{t i}^{s} \geq 0$ gives $p_{t i}^{s *} \geq \theta_{t i}^{s}$. As we are assuming that $q_{t i}^{*}=p_{t i}^{s *}$, then $q_{t i}^{*} \geq \theta_{t i}^{s}$ also holds. As $\theta_{t i}^{s} \leq p_{t i}^{s *}<\bar{P}_{i}$ definition (53) sets a value of $p_{t i}^{d, s}$ that will be either $\theta_{t i}^{s}$ or $\underline{P}_{i}$, depending whether $\theta_{t i}^{s}>\underline{P}_{i}$ or $\theta_{t i}^{s} \leq \underline{P}_{i}$ respectively. Nevertheless, in both cases $p_{t i}^{d, s} \leq q_{t i}^{*}$, and then $p_{t i}^{s *}=\max \left\{q_{t i}^{*}, p_{t i}^{d, s} \leq q_{t i}^{*}\right\}=q_{t i}^{*}$

(e) $\underline{P}_{i}=q_{t i}^{*}=p_{t i}^{s *}<\bar{P}_{i}$ : Condition (47) sets $\bar{\mu}_{t i}^{s}=0$ which, by taking into account equation (55) and $\hat{\mu}_{t i}^{s} \geq 0$, provides $p_{t i}^{s *}=\underline{P}_{i} \geq \theta_{t i}^{s}$. Then, by definition (53), $p_{t i}^{d, s}=\underline{P}_{i}$, and $p_{t i}^{s *}=\max \left\{q_{t i}^{*}=\underline{P}_{i}, p_{t i}^{d, s}=\underline{P}_{i}\right\}=\underline{P}_{i}$

Lemma 3 (Optimal matched energy, linear costs). Let $x^{*}$ be an optimal solution of the (DABFC) problem. Then for any unit $i$ with linear generation cost (i.e. $c_{i}^{q}=0$ ) committed at period $t$ (i.e. $\left.i \in I_{\text {on }}^{*}\right)$, the optimal value of the matched energy $p_{t i}^{s *}$ can be expressed as:

$$
p_{t i}^{s *}= \begin{cases}q_{t i}^{*} & \text { if } \lambda_{t}^{d, s} \leq c_{i}^{l} \\ \bar{P}_{i} & \text { if } \lambda_{t}^{d, s}>c_{i}^{l}\end{cases}
$$

Proof As lemma 1 sets forth, any optimal solution of the (DABFC) problem must satisfy the KKT system (43)-(51). When $c_{i}^{q}=0$ equation (43) can be expressed as:

$$
\hat{\mu}_{t i}^{s}-\bar{\mu}_{t i}^{s}=P^{s}\left(c_{i}^{l}-\lambda_{t}^{d, s}\right)
$$

with $P^{s}$ the probability of scenario $s$. There are three possible cases:

(a) $\lambda_{t}^{d, s}<c_{i}^{l}$ : in this case equation (58) implies that $\hat{\mu}_{t i}^{s}>\bar{\mu}_{t i}^{s}$ which gives rise to two different situations. In the first one $\hat{\mu}_{t i}^{s}>\bar{\mu}_{t i}^{s}>0$, that, together with equations (47) and (49) gives $p_{t i}^{s *}=q_{t i}^{*}=\bar{P}_{i}$. In the second one $\hat{\mu}_{t i}^{s}>\bar{\mu}_{t i}^{s}=0$ and the same KKT conditions forces $p_{t i}^{s *}=q_{t i}^{*} \leq \bar{P}_{i}$

(b) $\lambda_{t}^{d, s}>c_{i}^{l}$ : now equation (58) sets $\bar{\mu}_{t i}^{s}>\hat{\mu}_{t i}^{s}$, which again defines only two possibilities. In the first one the strict inequalities of $\bar{\mu}_{t i}^{s}>\hat{\mu}_{t i}^{s}>0$ hold and, considering equations (47) and (49), set $p_{t i}^{s *}=q_{t i}^{*}=\bar{P}_{i}$. In the second one, $\bar{\mu}_{t i}^{s}>\hat{\mu}_{t i}^{s}=0$ which, after equations (47) and (49), allows the matched energy to be expressed as $p_{t i}^{s *}=\bar{P}_{i} \geq q_{t i}^{*}$.

(c) $\lambda_{t}^{d, s}=c_{i}^{l}$ : in this case equation (58) gives $\hat{\mu}_{t i}^{s}=\bar{\mu}_{t i}^{s}$. Two cases must be analyzed here. In the first one, where $\hat{\mu}_{t i}^{s}=\bar{\mu}_{t i}^{s}=0$, the KKT system (43)-(51) doesn't impose any condition over the relation between $p_{t i}^{s *}, q_{t i}^{*}$ and the bound $\bar{P}_{i}$. Therefore, for a given optimal solution $x^{*}$ of the (DABFC) problem, any feasible value of the variables $p_{t i}^{s *}$ and $q_{t i}^{*}$ are equally optimal, in particular, the value $p_{t i}^{s *}=q_{t i}^{*}$. The second case to be analyzed is the case where $\hat{\mu}_{t i}^{s}$ and $\bar{\mu}_{t i}^{s}$ are both strictly positives. Then, equations (49) gives $p_{t i}^{s *}=q_{t i}^{*}$.

Lemmas 2 and 3 establish the expressions of the optimal matched energy variable for any spot price $\lambda_{t}^{d, s}$ at any optimal solution of the (DABFC) problem. The bid strategies consistent with such a matched energy will be developed in the next section. 


\subsection{Optimal bid function}

In section 2.4 the concepts of bid and matched energy functions were introduced. The matched energy function associated with a given bid function $\lambda_{t i}^{b}$ was defined as

$$
p_{t i}^{m}\left(\lambda_{t}^{d}\right) \stackrel{\text { def }}{=} \max \left\{p_{t i}^{b} \in\left[0, \bar{P}_{i}\right] \mid \lambda_{t i}^{b}\left(p_{t i}^{b}\right) \leq \lambda_{t}^{d}\right\}
$$

Assumption 1 supposes the existence of a bid function, coherent with the (DABFC) problem, in the sense expressed in the following definition:

Definition 3 (Bid functions's optimality conditions). Let $x^{* \prime}=\left[u^{*}, a^{*}, e^{*}, p^{*}, q^{*}, f^{*}\right]^{\prime}$ be an optimal solution of the (DABFC) problem. The bid function $\lambda_{t i}^{b *}$ of a thermal unit i committed at period $t$ (i.e. $i \in I_{o n_{t}}^{*}$ ) is said to be optimal w.r.t. the (DABFC) problem and solution $x^{*}$ if the value of the matched energy function associated with any scenario's clearing price $\lambda_{t}^{d, s}, p_{t i}^{m}\left(\lambda_{t}^{d, s}\right)$, coincides with the optimal matched energy $p_{t i}^{s *}$ given by expressions (52) and (57).

The equivalence $p_{t i}^{m}\left(\lambda_{t}^{d, s}\right) \equiv p_{t i}^{s *}$ assures us that, if a GenCo submits systematically optimal bid functions to the dayahead market, the expected value of the benefits will be maximized, as long as the actual behaviour of the clearing price $\lambda_{t}^{d}$ has been captured by the set of scenarios $S$. The next lemma develops the expression of the optimal bid function associated with the (DABFC) problem:

Lemma 4 (Optimal bid function). Let $x^{* \prime}=\left[u^{*}, a^{*}, e^{*}, p^{*}, q^{*}, f^{*}\right]^{\prime}$ be an optimal solution of the (DABFC) problem and $i$ any thermal unit committed on period $t$ at the optimal solution (i.e. $i \in I_{o n_{t}}^{*}$ ). Then:

(i) If the generation cost is quadratic convex, the bid function:

$$
\lambda_{t i}^{b q *}\left(p_{t i}^{b}\right)= \begin{cases}0 & \text { if } p_{t i}^{b} \leq q_{t i}^{*} \\ 2 c_{i}^{q} p_{t i}^{b}+c_{i}^{l} & \text { if } q_{t i}^{*}<p_{t i}^{b} \leq \bar{P}_{i}\end{cases}
$$

is optimal w.r.t. the (DABFC) problem and the optimum $x^{*}$.

(ii) If the generation cost is linear the bid function:

$$
\lambda_{t i}^{b l *}\left(p_{t i}^{b}\right)= \begin{cases}0 & \text { if } p_{t i}^{b} \leq q_{t i}^{*} \\ c_{i}^{l} & \text { if } q_{t i}^{*}<p_{t i}^{b} \leq \bar{P}_{i}\end{cases}
$$

is optimal w.r.t. the (DABFC) problem and the optimum $x^{*}$.

Proof We will consider first part (i) of the lemma. To illustrate the proof, the expression (59) has been represented graphically in Fig.2(a). It can be easily verified that the matched energy function associated with the bid function $\lambda_{t i}^{b q *}$ is (Fig.2(b)):

$$
p_{t i}^{m *}\left(\lambda_{t}^{d}\right)=\left\{\begin{array}{lll}
q_{t i}^{*} & \text { if } & \lambda_{t}^{d} \leq \lambda_{t i} \\
\left(\lambda_{t}^{d}-c_{i}^{l}\right) / 2 c_{i}^{q} & \text { if } & \lambda_{t i}<\lambda_{t}^{d} \leq \bar{\lambda}_{t i} \\
\bar{P}_{i} & \text { if } & \lambda_{t}^{d}>\bar{\lambda}_{t i}
\end{array}\right.
$$

where the threshold prices $\underline{\lambda}_{t i}$ and $\bar{\lambda}_{t i}$ are defined as:

$$
\underline{\lambda}_{t i}=2 c_{i}^{q} q_{t i}^{*}+c_{i}^{l} \quad ; \quad \bar{\lambda}_{t i}=2 c_{i}^{q} \bar{P}_{i}+c_{i}^{l}
$$

To prove the part (i) of the lemma it is only necessary to demonstrate that $p_{t i}^{m *}\left(\lambda_{t}^{d, s}\right) \equiv p_{t i}^{s *}$, where $p_{t i}^{s *}$ is the value of the optimal matched energy at scenario $s$, given by (52). First notice that, if $\lambda_{t}^{d}=\lambda_{t}^{d, s}$, the spot price at scenario $s$, then the matched energy function (61) can be rewritten as:

$$
p_{t i}^{m *}\left(\lambda_{t}^{d, s}\right)= \begin{cases}q_{t i}^{*} & \text { if } \quad \lambda_{t}^{d, s} \leq \lambda_{t i} \\ \theta_{t i}^{s} & \text { if } \quad \underline{\lambda}_{t i}<\lambda_{t}^{d, s} \leq \bar{\lambda}_{t i} \\ \bar{P}_{i} & \text { if } \quad \lambda_{t}^{d, s}>\bar{\lambda}_{t i}\end{cases}
$$

where $\theta_{t i}^{s}$ is the parameter defined in equation (54). Now, the equivalence $p_{t i}^{m *}\left(\lambda_{t}^{d, s}\right) \equiv p_{t i}^{s *}=\max \left\{q_{t i}^{*}, p_{t i}^{d, s}\right\}$ can be easily verified for the three cases of expression (63) (please, refer to Fig. 2(b) for a graphical interpretation of these three cases): 


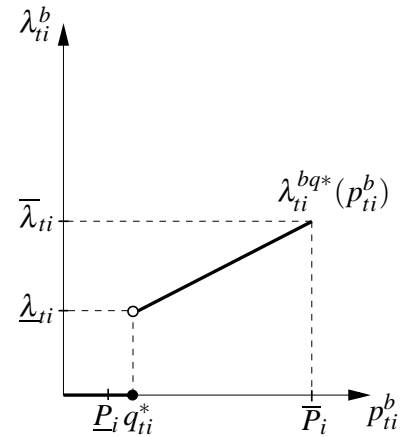

(a)

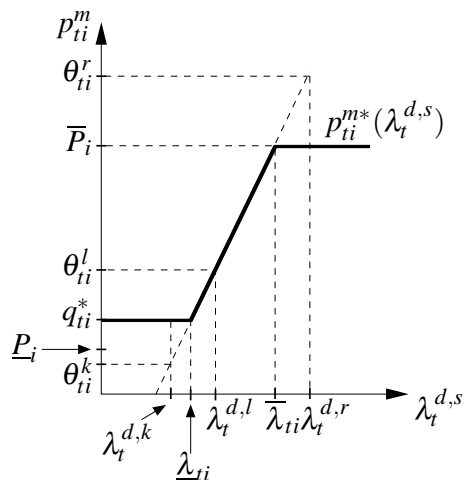

(b)

Figure 2: Optimal bid function $\lambda_{t i}^{b q *}\left(p_{t i}^{b}\right)$ (a) and associated matched energy function $p_{i t}^{m *}\left(\lambda_{t}^{d, s}\right)$ (b) for units with quadratic generation costs.

(a) If, for some $k \in S, \lambda_{t}^{d, k} \leq \underline{\lambda}_{t i}$ then $\theta_{t i}^{s} \leq q_{t i}^{*}$ and, by definition (53), $p_{t i}^{d, k}=\max \left\{\theta_{t i}^{k}, \underline{P}_{i}\right\}$, which will always be less than or equal to $q_{t i}^{*}$. Then, we can write that $p_{t i}^{m *}\left(\lambda_{t}^{d, k}\right)=q_{t i}^{*}=\max \left\{q_{t i}^{*}, p_{t i}^{d, k} \leq q_{t i}^{*}\right\}=p_{t i}^{k *}$.

(b) If, for some $l \in S, \underline{\lambda}_{t i}<\lambda_{t}^{d, l} \leq \bar{\lambda}_{t i}$ then $q_{t i}^{*}<\theta_{t i}^{l} \leq \bar{P}_{i}$ which, by definition (53), gives $p_{t i}^{d, l}=\theta_{t i}^{l}$ and $p_{t i}^{m *}\left(\lambda_{t}^{d, l}\right)=$ $\theta_{t i}^{l}=\max \left\{q_{t i}^{*}, p_{t i}^{d, l}=\theta_{t i}^{l}>q_{t i}^{*}\right\}=p_{t i}^{l *}$

(c) If, for some $r \in S, \lambda_{t}^{d, r}>\bar{\lambda}_{t i}$ then $\theta_{t i}^{r}>\bar{P}_{i}$ which, together with definition (53), sets $p_{t i}^{d, r}=\bar{P}_{i}$ and: $p_{t i}^{m *}\left(\lambda_{t}^{d, r}\right)=$ $\bar{P}_{i}=\max \left\{q_{t i}^{*}, p_{t i}^{d, r}=\bar{P}_{i}>q_{t i}^{*}\right\}=p_{t i}^{r *}$

To demonstrate the equivalence $p_{t i}^{m *}\left(\lambda_{t}^{d, s}\right) \equiv p_{t i}^{s *}$ when $c_{i}^{q}=0$ (part (ii) of the lemma), observe that the optimal matched energy function associated with the optimal bid function $\lambda_{t i}^{b l *}$ is:

$$
p_{t i}^{m *}\left(\lambda_{t}^{d}\right)= \begin{cases}q_{t i}^{*} & \text { if } \quad \lambda_{t}^{d} \leq c_{i}^{l} \\ \bar{P}_{i} & \text { if } \quad \lambda_{t}^{d}>c_{i}^{l}\end{cases}
$$

which is represented in Fig. 3(b). Expression (64) is equivalent to expression (57), and then, $p_{t i}^{m *}\left(\lambda_{t}^{d, s}\right) \equiv p_{t i}^{s *} \forall s \in S$

Observe that a direct result of lemma 4 is that assumption 1 always holds.

As mentioned before, the (DABFC) problem assures us that, if the optimal bids (59)-(60) are submitted to the DayAhead Market, the expected value of the benefit function $B$ (14)-(16) will be maximized. There are two important considerations about these optimal bid functions. The first one is that the optimal bid functions (59)-(60) represent to some extent a generalization of the classical self-commitment problem treated by several authors (Conejo et al. [6], Gountis and Bakirtzis [7]). Effectively, if the thermal unit $i$ does not contribute to covering futures contracts at period $t$ (i.e., $q_{t i}^{*}=0$ ), then the optimal bid function offers the complete production of the thermal plant $p_{t i}^{b}$ at its true marginal cost, $2 c_{i}^{q} p_{t i}^{b}+c_{i}^{l}$ or $c_{i}^{l}$ depending on the generation costs functions. Second, the true bid function required by the market's operator in the MIBEL is a stepwise non-decreasing function. The optimal bid function (60) satisfies this condition, but (59) is not stepwise. This is an approximation commonly adopted in the literature (see Gountis and Bakirtzis [7]) and does not represent a serious limitation on the practical interest of the model, because it is always possible to built a posteriori a stepwise approximation of the resulting optimal bid (59).

\section{Numerical examples}

The model (17)-(26) has been tested with real data of a Spanish GenCo and MIBEL market prices. The model has been implemented in AMPL (Fourer et al. [27]) and solved with CPLEX [28] using a SunFire X2200 with two dual core AMD Opteron 2222 processors at $3 \mathrm{GHz}$ and $32 \mathrm{~Gb}$ of RAM memory. 


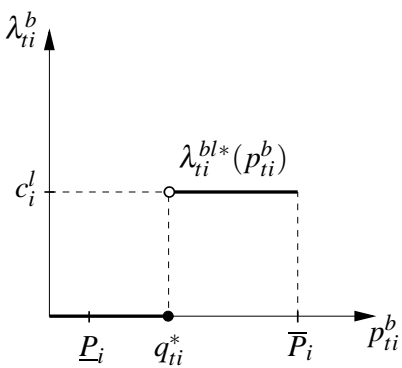

(a)

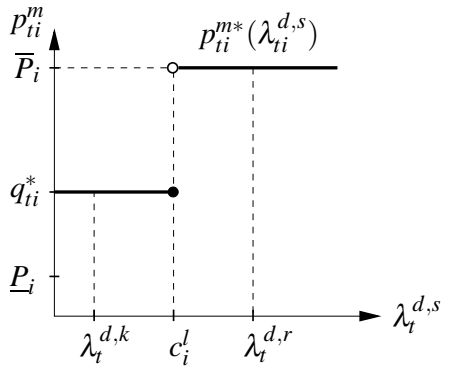

(b)

Figure 3: Optimal bid function $\lambda_{t i}^{b q *}\left(p_{t i}^{b}\right)$ (a) and associated matched energy function $p_{t i}^{m *}\left(\lambda_{t}^{d, s}\right)$ (b) for units with linear generation costs.

\begin{tabular}{cccccccc}
\hline$t$ & $\begin{array}{c}c_{t}^{b} \\
€\end{array}$ & $\begin{array}{c}c_{t}^{l} \\
€ / \mathrm{MWh}\end{array}$ & $\begin{array}{c}c_{t}^{q} \\
€ / \mathrm{MWh}^{2}\end{array}$ & $\begin{array}{c}\underline{p}_{t} \\
\mathrm{MW}\end{array}$ & $\begin{array}{c}\bar{p}_{t} \\
\mathrm{MW}\end{array}$ & $\begin{array}{c}c_{t}^{\text {on }} \\
€\end{array}$ & $\begin{array}{c}c_{t}^{\text {off }} \\
€\end{array}$ \\
\hline 1 & 151.08 & 40.37 & 0.015 & 160.0 & 350.0 & 412.80 & 412.80 \\
2 & 554.21 & 36.50 & 0.023 & 250.0 & 563.2 & 803.75 & 803.75 \\
3 & 97.56 & 43.88 & 0.000 & 80.0 & 284.2 & 244.80 & 244.80 \\
4 & 327.02 & 28.85 & 0.036 & 160.0 & 370.7 & 438.40 & 438.40 \\
5 & 64.97 & 45.80 & 0.000 & 30.0 & 65.0 & 100.20 & 100.20 \\
6 & 366.08 & -13.72 & 0.274 & 60.0 & 166.4 & 188.40 & 188.40 \\
7 & 197.93 & 36.91 & 0.020 & 160.0 & 364.1 & 419.20 & 419.20 \\
8 & 66.46 & 55.74 & 0.000 & 110.0 & 313.6 & 1298.88 & 1298.88 \\
9 & 372.14 & 105.08 & 0.000 & 90.0 & 350.0 & 1315.44 & 1315.44 \\
\hline
\end{tabular}

Table 1: Operational characteristics of the thermal units

\subsection{Data sources}

All the data of this work is public and it has been either downloaded directly from the indicated web pages or calculated by using some other public data. The sources for all data used in the case studies are:

- Market data: the Day-Ahead Market price has been available at OMEL's site (OMEL [25]) since January 1998 until today. In this work we use the data from January $1^{\text {st }}, 2004$ to May $30^{\text {th }}, 2009$. Generic data about the quantities and clearing prices of the FCs is available at OMIP's site (OMIP [24]), this data has been used to define some examples of FCs.

- Generation Company data: the information about the thermal units in the study belongs to a GenCo that bids daily in the Day-Ahead Market and also participates in the Derivatives Market (Table 1). Most of the information about the generation units is available at the CNE's site (CNE [29]).

\subsection{Construction of the set of scenarios}

The optimization model presented in this work is stochastic due to the presence of a random variable, the DayAhead Market price (see Section 2). This random variable has the characteristics of a financial time series and, in order to be introduced in the stochastic model, it has to be discretized on a scenario tree. In particular, the model presented in this work is a two-stage stochastic problem and, for this kind of model, a set of individual scenarios with its corresponding probabilities is sufficient. In this work, we have observed the following steps in order to obtain the required scenario set:

1. Time series model: the Spanish Day-Ahead Market price presents the following characteristics: high frequency, nonconstant mean and variance, multiple seasonality, calendar effect, high volatility and high presence of picks 


\begin{tabular}{ccccc}
\hline$|S|$ & c.v. & CPU(s) & E(benefits) $(€)$ & $\frac{\left\|x^{s}-x^{150}\right\|}{\left\|x^{150}\right\|}$ \\
\hline 10 & 3360 & 13 & 1350830 & 0.3350 \\
20 & 5760 & 55 & 1085240 & 0.2997 \\
30 & 8160 & 112 & 1093900 & 0.2913 \\
40 & 10560 & 216 & 1081010 & 0.1821 \\
50 & 12960 & 444 & 1107110 & 0.1764 \\
75 & 18960 & 2100 & 1087860 & 0.0712 \\
100 & 24960 & 3319 & 1089280 & 0.0712 \\
150 & 36960 & 4244 & 1084880 & \\
\hline \multicolumn{5}{c}{$|T|=24 ;|I|=9 ;$ b.v. $=720$} \\
\hline \multicolumn{5}{c}{}
\end{tabular}

Table 2: Results for different number of scenarios

(Nogales et al. [30]), which are the common characteristics of a financial time series. The market price has been characterized by an auto-regressive integrated moving average model. We work with the log scale of the price in order to avoid the nonconstant variance, specifically:

$$
\ln \left(\lambda^{d}\right) \sim \operatorname{ARIMA}(5,0,2)(8,0,1)_{24}(3,0,3)_{168}
$$

The model is fitted based on the data from 2004 to 2007. The coefficients are estimated by maximum likelihood estimation.

2. Scenario generation: one of the most usual mechanisms for this discretization is the simulation of prices scenarios for the day in study Kaut and Wallace [31]. Thus, once the model has been fitted we generate 300 simulated scenarios for the 24 hours of the day in study.

3. Scenario reduction: a set of decision variables is required for each scenario, so the reduction of the number of scenarios will reduce the dimension of the problem and ease the computational resolution. Following the algorithm described in Growe-Kuska et al. [32], the set of scenarios has been reduced preserving at maximum the characteristics of the simulated set.

In stochastic programming models, the number of scenarios is a critical decision. We deal with this problem by increasing the number of scenarios until the stabilization of the objective function optimal value. The original tree has 300 scenarios that have been reduced to sets of 150, 100, 75, 50, 40, 30, 20 and 10 scenarios. In table 2 the main parameters of each test are summarized: number of scenarios (S), number of continuous variables (c.v.), CPU time in seconds (CPU(s)), the value of the expected benefits (E(benefits) $(€)$ ), and the difference in the first stage variables value between the reduced set and the one with 150 scenarios, given in fraction of unit $\frac{\left\|x^{s}-x^{150}\right\|}{\left\|x^{150}\right\|}$ where $x^{s}=\left[q^{*}, u^{*}\right]^{\prime}$ $\forall s \in S$ ); the number of binary variables (b.v.) is independent of the number of scenarios and it is equal to 720 for all instances. The value of E(benefits) only considers the benefit from the Day-Ahead Market (terms (16) and (15)), ignoring the constant FCs income (14), and corresponds to minus the objective function of the (DABFC) problem. It can be observed how the CPU time increases with the number of scenarios because of the proportional relationship between them and the number of continuous variables (the number of binary variables is independent of the number of scenarios). It can be seen also the stabilization of the value of the objective function when the number of scenarios grows (Fig. 4(a)) and the convergence to zero of the difference in the optimal value of the first stage decision variables between each reduced set and the largest one (Fig. 4(b)). Both values converge from approximately 75 scenarios and the computational time is acceptable. Any increase in the number of scenarios from 75 to 100 does not improve the optimal solution accuracy enough to justify the $50 \%$ increase in the CPU time. Therefore 75 will be the selected number of scenarios for the computational tests.

\subsection{Computational results}

A set of computational tests has been performed in order to validate the proposed modeling of the day-ahead bid with FCs problem. The instances used in the test have 3 bilateral contracts, 9 thermal units and 24 hourly. The computational tests are done changing the quantity of energy allocated to physical FCs in order to study its influence 

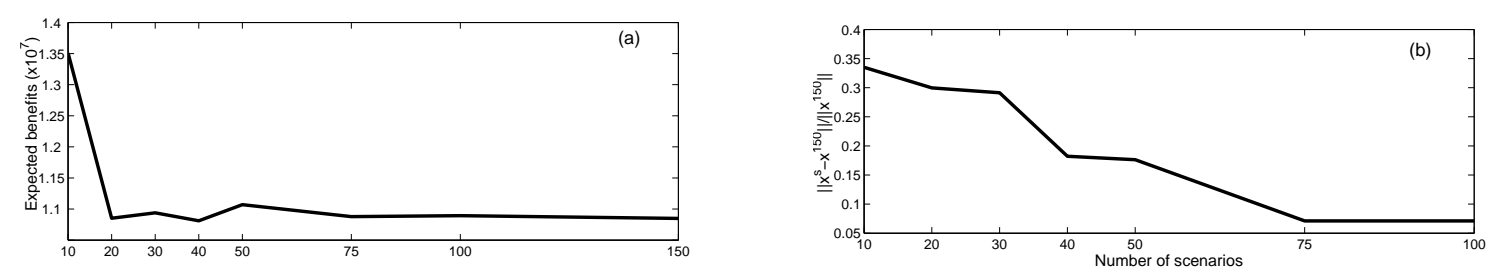

Figure 4: (a) Expected benefits for each reduced set of scenarios (b) First stage variables convergence evaluated as $\frac{\left\|x^{s}-x^{150}\right\|}{\left\|x^{150}\right\|}, x^{s}=\left[q^{*}, u^{*}\right]^{\prime} \forall s \in S$

$$
\begin{array}{cc}
\overline{ } \bar{P} & \mathrm{E} \text { (benefits) } \\
\hline 5 & 1823170 \\
40 & 1107110 \\
75 & -2800460 \\
\hline 10 ;|S|=24 ;|I|=75 ; \text { c.v. }=720 ; \text { b.v. }=12960
\end{array}
$$

Table 3: Dependency of the Day-Ahead Market benefits with the fraction of the total generation capacity allocated to futures contracts.

in the results. The status of the units before the first interval is fixed as all open, allowing them to be closed or remain opened at hour 1; this is done in order to give more freedom to the unit commitment.

The quantity allocated to FCs is confidential and therefore there is no real public data for the units in the study. The set of computational tests presented is based on the percentage of the total energy generation capacity that the GenCo has allocated in FCs, $\% \bar{P}=\sum_{\forall j \in F} L_{j} / \sum_{\forall i \in I} \bar{P}_{i}$. For this case study, we include the 9 available units distributed in one or more of the 3 UCPs created, each of them corresponding to one FC. In table 3 the main parameters of the computational test are summarized for three different values of $\% \bar{P}: 5 \%, 40 \%$ and $75 \%$. The computational time for the 3 cases is approximately the same but the value of the expected benefits differs. Observe that when $\% \bar{P}=75 \%$ the GenCo experiences a loss in the Day-Ahead Market, which should be compensated with the FCs incomes (14).

Figure 5 shows the optimal bid function for unit 1 at interval $12, \lambda_{12,1}^{b q *}\left(p_{12,1}^{b}\right)$ (sec. 3, equation (59)), for the different values of $\% \bar{P}$ considered. The plot represents an adaptation of the optimal bid function provided by the model to the real bid function that the GenCo has to submit to the MIBEL Day- Ahead Market operator. This real bid function is composed of ten pairs (energy, price) with increasing price (points (a)) that can be represented as a stepwise increasing curve starting at the point defined by the instrumental price offer $\left(q_{12,1}^{*}, 0\right)$. The following steps are constructed by following the optimal bid function, in a way that the coordinates of the points (a) are $\left(p_{12,1}^{b}, \lambda_{12,1}^{b q *}\left(p_{12,1}^{b}\right)\right)$, with the values of the bid energy $p_{12,1}^{b}$ evenly distributed between $q_{12,1}^{*}$ and $\bar{P}_{12}$. Notice that for the first case (solid line) the unit has no energy allocated to FCs so the instrumental offer's energy is the minimum operational limit (160MW) because, as the unit is committed, the matched energy has to be at least this quantity. For the other two cases the energy allocated to FCs is $186 \mathrm{MW}$ (dotted line) and 256MW (dashed line). In the following analysis, the percentage of available energy used for physical FCs will be fixed at $40 \%$.

Figure 6(a) shows variable $q_{t i}^{*}$, the instrumental price bid, energy for each unit and interval. The values shown in the ordinate axis are the minimum and maximum power capacity of each unit. This instrumental price bid can be either the quantity allocated to FCs or the minimum operational limit of the unit. Fig. 6(a) also represents the unit commitment, because if the unit is not producing the minimum operational limit it means the unit is off. We can see that unit 5 starts-up at 10 a.m. and units 2, 8 and 9 start-up after 6 p.m. This behavior is related to the prices structure because in the MIBEL the highest prices are at noon and in the evening, the peak hours being after 6 p.m.

Figure 6(b) represents variable $f_{t i j}^{*}$, the optimal economic dispatch of each FC. This representation shows how the contract is settled among the different units of each UCP. Three kinds of physical FCs have been considered, 200 MWh in a weekly contract, $500 \mathrm{MWh}$ in a monthly contract and $500 \mathrm{MWh}$ in a yearly contract. It can be observed that every unit of a given UCP contributes to the corresponding FC in at least one interval. Notice how in the off-peak hours (lower clearing prices), if possible, each contract is settled by the cheapest unit in the UCP, for example unit 7 


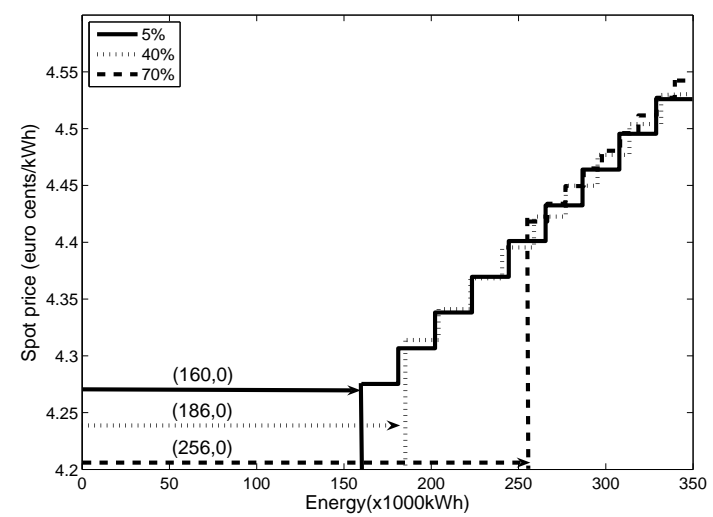

Figure 5: Optimal offer for unit 1 at hour 12
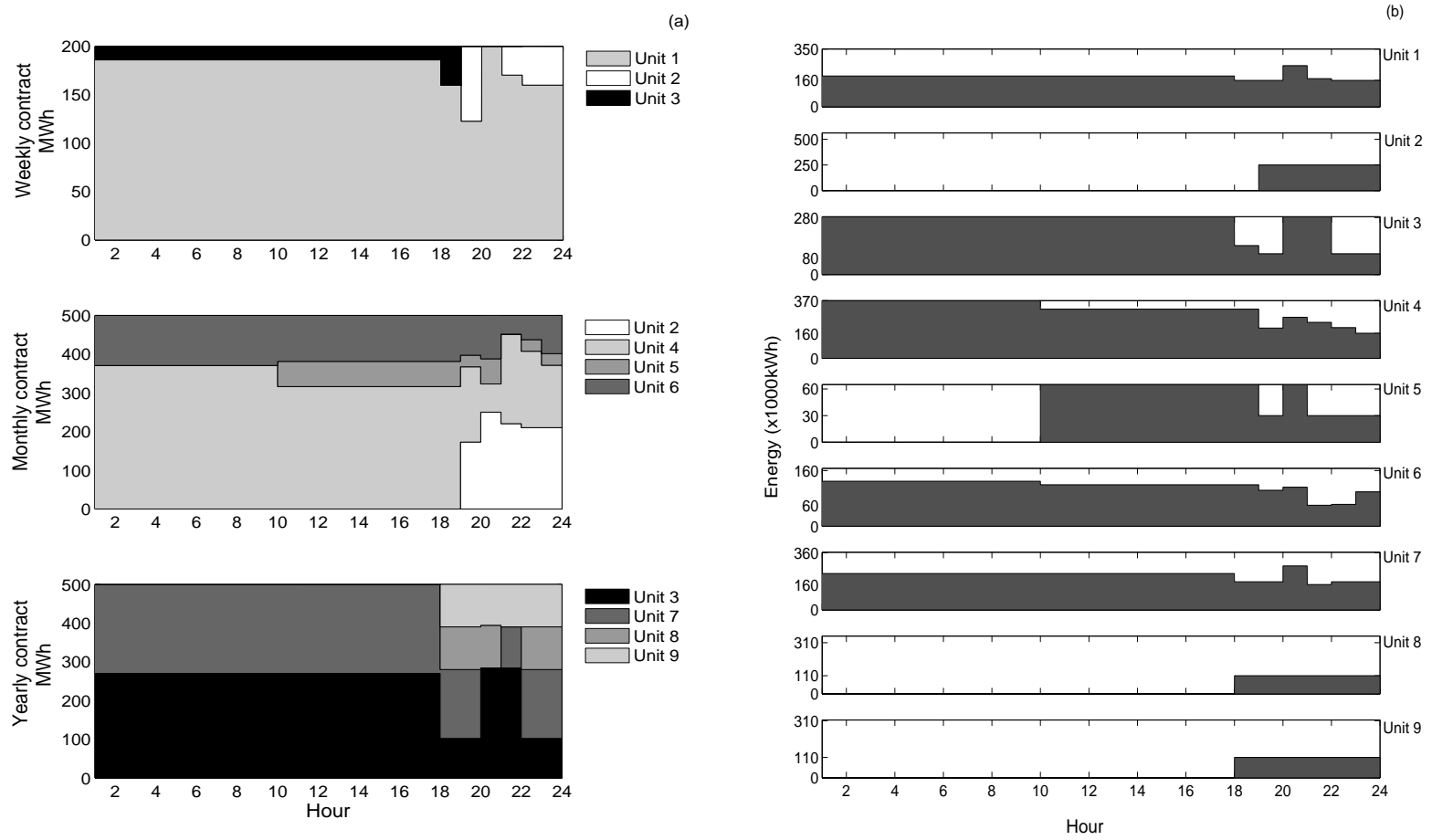

Figure 6: (a) Optimal instrumental price bid energy $q_{i t}^{*}$ for each unit and interval (b) Economic dispatch of each futures contracts, $f_{i t j}$ 

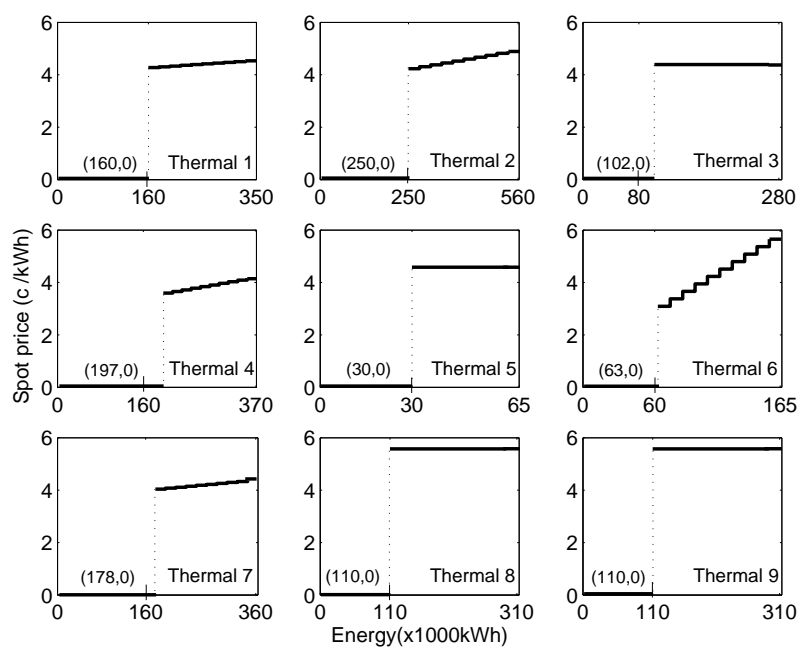

Figure 7: Bidding curve for each unit at hour 23

in the yearly contract or unit 6 in the monthly contract. Specifically, as unit 7 cannot generate all the energy needed for the yearly contract, unit 3 has to contribute covering the rest of the contract. For this reason the weekly contract is not fully covered by unit 3 , which is the cheapest one, but by unit 1 , since unit 3 is generating for the yearly contract. In the case of the monthly contract, since the maximum power capacity of unit 6 is insufficient, the contract must be covered with the help of the next cheapest unit, unit 4. The results of the peak hours are not as easily interpretable because Day-Ahead Market incomes are greater and its relation with production costs allows all the units to participate both in FCs and day-ahead bidding.

Figure 7 shows the optimal bid curves for each committed thermal unit at hour 12. The numerical values shown in the abscissa axis indicate the minimum and maximum power capacity. The first interval is always the instrumental price bid, which is indicated in parenthesis as (price, quantity). Units 3,5 and 9 have linear generation costs and its real bid coincides with the optimal bid function $\lambda_{t i}^{b l *}$ expressed in equation (60). The rest of the units have quadratic generation costs and the function represented corresponds to the adaptation of the optimal bid functions $\lambda_{t i}^{b q *}$ (equation (59) to the real stepwise bid function built as in figure 5. Notice that there are some thermal units that have $q_{t i}$ greater than the minimum power capacity, specifically units 3, 4, 6 and 7, this fact is a direct consequence of the participation of these units in the FC being covered.

In summary, this results give the GenCo the optimal bidding strategy following the market operator rules. The main difference with other bidding strategies is that the optimal value of $q_{t i}$ corresponds directly to the optimal zero price bid, that is to say, the first step of the step-wise bidding curve.

\section{Conclusions}

This work has developed a new quadratic mixed-integer stochastic programming model, for the optimal DayAhead Bid with Future Contracts problem (DABFC). The optimal solution of our model determines the unit commitment of the thermal units, the optimal instrumental price bidding strategy for the generation company and the economic dispatch of the committed FCs for each hour so as to maximize the benefits arising from the Day-Ahead Market while satisfying the thermal operational constraints and the MIBEL's rules concerning the integration of the energy of the Physical Futures Contract in the Day-Ahead Market. As a result of the study of the properties of the optimal solution of the (DABFC) problem, the proposed model also provides the analytical expression of the optimal bid functions that ensures the maximization of the long run expected benefits. The expression for the optimal bid functions represents a generalization of the marginal cost bid function for those utilities that must integrate the settled 
energy of the physical FCs within their bid to the Day-Ahead Market obeying the MIBEL regulation. The model was implemented and solved with real data of MIBEL market prices and a Spanish generation company with participation in the Derivatives and Day-Ahead Markets. The results of the computational tests validate the model and show the influence in the optimal bidding strategy of the generation company of the participation in physical futures contract.

\section{A. Notation}

\section{A.1. Parameters}

$c_{t}^{b}, c_{t}^{l}, c_{t}^{q}$ : constant, linear and quadratic coefficients of the generation cost function of unit $t$.

$c_{t}^{o f f}, c_{t}^{o n}$ : shut-down and start-up cost of unit $t$.

$F$ : set of futures contracts.

$F_{t}$ : set of futures contracts in which unit $t$ participates.

$L_{j}$ : due settled energy of contract $j$.

$\lambda^{d}=\left\{\lambda_{1}^{d}, \ldots, \lambda_{i}^{d}, \ldots, \lambda_{I}^{d}\right\}$ : clearing prices of the $|I|$ Day-Ahead Markets.

$\lambda^{d, s}=\left\{\lambda_{1}^{d, s}, \ldots, \lambda_{i}^{d, s}, \ldots, \lambda_{I}^{d, s}\right\}$ : clearing prices of the $|I|$ Day-Ahead Markets for scenario $s$.

$\lambda_{j}^{f}$ : settlement price of futures contract $j$.

$\underline{\lambda}_{i t}, \bar{\lambda}_{i t}$ : threshold prices used in the definition of the optimal matched energy function $p_{i t}^{m *}$.

$\min _{t}^{\text {off }}, \min _{t}^{\text {on }}$ : operational minimum idle and in service time of unit $t$.

$P^{s}=P\left[\lambda^{d}=\lambda^{d, s}\right]$ : probability of scenario $s$.

$\underline{P}_{t}, \bar{P}_{t}$ : lower and upper bound on the energy generation of a committed unit $t$.

$p_{i t}^{d, s}:$ auxiliary parameter used in the definition of the optimal matched energy $p_{i t}^{s *}$ of unit $t$ at interval $i$ and scenario $s$.

$S$ : set of scenarios.

$T$ : set of thermal units.

$T_{j}$ : set of thermal units that participates in contract $j$.

$T_{o n_{i}}^{*}$ : set of committed units at interval $i$ over the optimal solution.

$\theta_{i t}^{s}$ : auxiliary parameter used in the definition of the optimal matched energy $p_{i t}^{s *}$ of unit $t$ at interval $i$ and scenario $s$.

\section{A.2. Variables and multipliers}

$a_{i t}$ : binary variable indicating the shutting-down of unit $t$ at interval $i$

$e_{i t}$ : binary variable to indicate the turning-on of unit $t$ at interval $i$.

$u_{i t}$ : binary variable representing the on-off operating status of the unit $t$ at interval $i$.

$f_{i t j}$ : continuous variable representing the energy of the future contract $j$ allocated to thermal unit $t$ at interval $i$.

$p_{i t}^{s}$ : continuous variable of scenario $s$ for the matched energy of unit $t$ at interval $i$.

$q_{i t}$ : continuous variable standing for the energy of the instrumental price offer of unit $t$ at interval $i$

$\pi$ : Lagrange multiplier of the future contracts energy dispatching constraints (37).

$\tilde{\mu}$ : Lagrange multiplier of the instrumental price offer constraints (38).

$\bar{\mu}, \hat{\mu}$ and $\underline{\mu}$ : Lagrange multipliers of the bounding and coupling constraints (39)-(41).

$\breve{\mu}$ : Lagrange multipliers of the non-negativity of variables $f$.

\section{A.3. Functions}

B: Day-Ahead and Futures Market benefit function.

$\lambda_{i t}^{b}\left(p_{i t}^{b}\right)$ : bid function of unit $t$ at the $i^{t h}$ spot market.

$\lambda_{i t}^{b q *}\left(p_{i t}^{b}\right), \lambda_{i t}^{l q *}\left(p_{i t}^{b}\right)$ : optimal bid function at the $i^{t h}$ spot market for a unit $t$ with quadratic generation cost.

$p_{i t}^{m}\left(\lambda_{i}^{d}\right)$ : matched energy function providing the matched energy of unit $t$ at interval $i$ associated to a given bid function $\lambda_{i t}^{b}$ for a fixed clearing price $\lambda$.

$p_{i t}^{m *}\left(\lambda_{i}^{d}\right)$ : matched energy function associated to the optimal bid function $\lambda_{i t}^{b q *}$ or $\lambda_{i t}^{b l *}$.

Units: costs and prices are in $€ / M W h$ and energy terms in MWh. 


\section{References}

[1] S. J. Deng, S. S. Oren, Electricity derivatives and risk management, Energy 31 (6-7) (2006) 940-953.

[2] OMEL, Reglas de funcionamiento del mercado de produccin de energa elctrica. Anexo. BOE n.155. (in Spanish), 2007.

[3] G. B. Shrestha, S. Kai, L. Goel, An efficient stochastic self-scheduling technique for power producers in the deregulated power market, Electric Power Systems Research 71 (1) (2004) 91-98.

[4] C. Triki, P. Beraldi, G. Gross, Optimal capacity allocation in multi-auction electricity markets under uncertainty, Computers \& Operations Research 32 (2) (2005) 201-217.

[5] R. Musmanno, N. Scordino, C. Triki, A. Violi, A multistage formulation for generation companies in a multi-auction electricity market, IMA Journal of Management Mathematics doi:10.1093/iamamn/dpp008.

[6] A. J. Conejo, F. J. Nogales, J. M. Arroyo, Price-Taker Bidding Strategy Under Price Uncertainty, IEEE Transactions on Power Systems 17 (4) (2002) 1081-1088.

[7] V. P. Gountis, A. G. Bakirtzis, Bidding Strategies for Electricity Producers in a Competitive Electricity Marketplace, IEEE Transactions on Power Systems 19 (1) (2004) 356-365.

[8] N. Nabona, A. Pages, A three-stage short-term electric power planning procedure for a generation company in a liberalized market, International Journal of Electrical Power \& Energy Systems 29 (5) (2007) 408-421.

[9] E. Ni, P. B. Luh, S. Rourke, Optimal integrated generation bidding and scheduling with risk management under a deregulated power market, IEEE Transactions on Power Systems 19 (2004) 600-609.

[10] M. P. Nowak, R. Schultz, M. Westphalen, A Stochastic Integer Programming Model for Incorporating Day-Ahead Trading of Electricity into Hydro-Thermal Unit Commitment, Optimization and Engineering 6 (2) (2005) 163-176.

[11] S.-E. Fleten, T. K. Kristoffersen, Stochastic programming for optimizing bidding strategies of a Nordic hydropower producer, European Journal of Operational Research 181 (2) (2007) 916-928.

[12] E. J. Anderson, A. B. Philpott, Optimal offer construction in electricity markets, Mathematics of Operations Research 27 (1) (2002) 82-100.

[13] E. J. Anderson, H. Xu, Necessary and sufficient conditions for optimal offers in electricity markets, SIAM Journal on Control and Optimization 41 (4) (2002) 1212-1228.

[14] J. C. Hull, Options, Futures and Other Derivatives, Prentice-Hall International, 5th edn., 2002.

[15] R. A. Collins, The Economics of Electricity Hedging and a Proposed Modification for the Futures Contracts for Electricity, IEEE Transactions on Power Systems 17 (1) (2002) 100-107.

[16] A. Neuberger, Hedging long-term exposures with multiple short-term futures contracts, The Review of Financial Studies 12 (3) (1999) 429-459.

[17] D. W. Carlton, Futures markets: their purpose, their history, their growth, their successes and failures, The Journal of Futures Markets 4 (3) (1984) 237-271.

[18] R. J. Kaye, H. R. Outhred, C. H. Bannister, Forward Contracts for the Operation of an Electricity Industy under Spot Pricing, IEEE Transactions on Power Systems 5 (1) (1990) 46-52.

[19] R. Bjorgan, C.-C. Liu, J. Lawarre, Financial Risk Management in a Competitive Electricity Market, IEEE Transactions on Power Systems 14 (4) (1999) 1285-1291.

[20] X. Chen, Y. He, Y. H. Song, Y. Nakanishi, C. Nakahishi, S. Takahashi, Y. Sekine, Study of impacts of physical contracts and financial contracts on bidding strategies of GenCos, Electrical Power \& Energy Systems 26 (2004) 715-723.

[21] A. J. Conejo, R. Garca-Bertrand, M. Carrin, A. Caballero, A. de Andrs, Optimal Involvement in Futures Markets of a Power Producer, IEEE Transactions on Power Systems 23 (2) (2008) 703-711.

[22] X. Guan, J. Wu, F. Gao, G. Sun, Optimization-Based Generation Asset Allocation for Forward and Spot Markets, IEEE Transactions on Power Systems 23 (4) (2008) 1796-1807.

[23] E. Tanlapco, J. Lawarre, C.-C. Liu, Hedging with Futures Contrats in a Deregulated Electricity Industry, IEEE Transactions on Power Systems 17 (3) (2002) 577-582.

[24] OMIP, Iberian Electricity Market: Derivatives Market Operator. http://www.omip.pt, 2008.

[25] OMEL, Iberian Electricity Market: Day-Ahead Market Operator. http://www.omel.es, 2008

[26] D. G. Luenberger, Linear and nonlinear programming, Kluwer Academic Publishers. Boston, 2nd edn., 2004.

[27] R. Fourer, D. M. Gay, B. W. Kernighan, AMPL: A modeling language for mathematical programming, CA: Brooks/Cole-Thomson Learning, 2nd edn., 2003.

[28] CPLEX, CPLEX Optimization subroutine library guide and reference. Version 11.0, cPLEX Division, ILOG Inc., Incline Village, NV, USA, 2008.

[29] CNE, Energy regulator of Spain. http://www.cne.es, 2008.

[30] F. J. Nogales, J. Contreras, A. J. Conejo, R. Espinola, Forecasting next-day electricity prices by time series models, Power Systems, IEEE Transactions on 17 (2) (2002) 342-348.

[31] M. Kaut, S. W. Wallace, Evaluation of Scenario-Generation Methods for Stochastic Programming, SPEPS, Working Paper 14, 2003.

[32] N. Growe-Kuska, H. Heitsch, W. Romisch, Scenario reduction and scenario tree construction for power management problems, in: IEEE Power Tech Conference Proceedings, Bologna, Italy, vol. 3, 7pp.Vol.3, 2003. 